\title{
Short-term Outcome after Posterior versus Lateral Surgical APPROACH FOr TOTAL Hip ARTHROPLASTY - A RANDOMized Clinical TRIAL*
}

\author{
W.-C. Witzleb ${ }^{1,2}$, L. Stephan ${ }^{1}$, F. Krummenauer ${ }^{3}$, A. Neuke, K.-P. Günther ${ }^{1}$ \\ ${ }^{1}$ Department of Orthopedic Surgery (Head of the department: Prof. Dr. K.-P. Günther), University Hospital Carl Gustav Carus, \\ Medical Falculty of the Technical University of Dresden, \\ ${ }^{2}$ Department of Orthopedic Surgery, Hospital St. Joseph- Stift, Health Forum Dresden, \\ ${ }^{3}$ Clinical Epidemiology and Health Economy Unit at the Orthopedic Surgery Department (Head of the unit: Prof. Dr. F. \\ Krummenauer), University Hospital Carl Gustav Carus, Medical Falculty of the Technical University of Dresden, \\ ${ }^{4}$ Department of Orthopedics and Rehabilitation (Head of the department: PD Dr. W. Schleicher), Bavaria- Klinik Kreischa, Germany
}

\begin{abstract}
Purpose: Currently, total hip replacement (THR) is most commonly performed via a posterior or a direct lateral approach, but the impact of the latter on the invention's outcome has yet not been quantified.

Methods: We compared the short-term outcome of cementless THR using the both approaches in a prospective, randomized controlled trial. 60 patients with unilateral osteoarthritis were included. Outcome assessment was performed one day before surgery and one week, four weeks, six weeks and 12 weeks after surgery, respectively, using the Harris Hip score as primary objective.

Results: We found no significant difference in the intraindividual Harris Hip Score improvement at the pre- and three months post-operative assessments between both treatment groups $(p=0.115)$. However, Harris Hip scores and most functional and psychometric secondary endpoints showed a consistent tendency of a slightly better three months result in patients implanted via the posterior approach. In contrast a significant shorter operating time of the direct lateral approach was recorded (67 minutes versus 76 minutes, $\mathrm{p}<0.001)$.

Conclusion: In our opinion this slightly better shortterm functional outcome after posterior approach is not clinical relevant. However, to make definitive conclusions all clinical relevant factors (i.e. mid- to longterm function, satisfaction, complication rates and long-term survival) have to be taken into account.

Level of evidence: I - therapeutic
\end{abstract}

Key words: Hip replacement, surgical approach, shortterm result, functional result

\section{INTRODUCTION}

Many different surgical approaches to the hip joint have been described. Currently, total hip replacement (THR) is most commonly performed via a posterior or a direct lateral approach [5].

The posterior approach is considered to be associated with less problems regarding gait, since the ab- ductor muscles are not dissected and damage to the superior gluteal nerve is very unlikely $[1,7]$. However, cup positioning is often more difficult and increased rates of dislocation have been reported [16, 20].

Critics of the direct lateral approach suggest that the violation of the hip abductors may lead to delay in recovery of abductor strength and late Trendelenburg gait $[9,18]$. The advantage proposed is the good exposure of the acetabulum, facilitating cup positioning which may decrease rates of dislocation and the decreased risk of sciatic nerve injury which is not close to the operative field.

The relative merits of these approaches have been widely debated in the orthopedic community. However, the limited number of studies as well as the limited reporting of their outcome measures prevents definitive conclusions to be drawn [12].

We therefore investigated the short-term outcome of patients undergoing total hip replacement via posterior versus direct lateral surgical approach in a prospective randomized manner. Our hypothesis was that the posterior approach would result in superior functional outcome after THR due to minor violation of the hip abductors.

\section{PATIENTS AND Methods}

\section{STUDY DESIGN:}

A prospective 1:1 randomization scheme was implemented (random numbers were generated by means of a block permutation algorithm) to allocate a total of 60 patients onto the treatment alternatives at hand (posterior versus lateral approach). Outcome assessment was performed one day before surgery and one week, four weeks, six weeks and 12 weeks after surgery, respectively, for each study patient. All assessments and their documentation were done

\footnotetext{
* The content of this manuscript is part of the respective Doctoral Theses of Mr Lars Stephan and Ms Anett Neuke (Medical Faculty, Technical University of Dresden).
} 
by two independent clinical investigators (A.N. and L.S.).

\section{PATIENT COHORT}

After approval of the study protocol by the local independent Ethics Committee, all patients between 45 and 65 years of age, who suffered from unilateral osteoarthritis of the hip and were admitted to our department for a primary cementless THR between October 2003 and February 2006, were screened for study inclusion. Exclusion criteria were age (older than 65 or younger than 45 years), known or suspected osteopenia or osteoporosis, deep infection or tumor illness of the hip, rheumatoid arthritis or higher grade developmental dysplasia of the hip (DDH Crowe stage II or higher), Charnley class $\mathrm{B}$ and $\mathrm{C}$ patients, previous operation or fracture of the joint, body mass index (BMI) over $40 \mathrm{~kg} / \mathrm{m}^{2}$, psychiatric illness and drug or alcohol abuse $[6,8]$. In addition, all patients who underwent Arthroplasty with other implants than stemmed THR (i.e. surface replacement) were excluded.

Written informed consent was obtained from all patients; patient information included detailed explanation of the two treatment alternatives under investigation and of the concept of randomization for treatment allocation. Afterwards, the patients were allocated into the two treatment groups at the day before surgery by means of a 1:1 block randomization scheme. At the day before surgery, additional standard patient information about details of the surgery at hand was communicated to each individual patient by the surgeon.

The treatment groups did not differ in the side of surgery (16 posterior versus 18 direct lateral approaches were done on the right hip) nor in their diagnosis (posterior group: 15 primary osteoarthritis cases, 12 DDH Crowe stage I, 1 protrusio acetabuli, 1 Perthes disease and 1 Epiphysiolysis capitis femoris, respectively; direct lateral group: 19 primary osteoarthritis cases, 7 DDH Crowe stage I, 2 protrusio acetabuli, 1 Perthes disease, 1 AVN, respectively). $70 \%$ of the hips of the posterior group showed a radiographic arthritis grade $4,30 \%$ showed a grade 3 according to the Kellgren and Lawrence scoring system [13]. The direct laterally approached patients were graded 4 in $80 \%$ and 3 in $20 \%$, respectively.

The distributions of gender, age and BMI did not significantly differ between the patients groups: The posterior group showed a median age of 55 years (47 - 64 years) versus 58 years $(46-64$ years) in the direct lateral group (Wilcoxon $\mathrm{p}=0.227$ ), the samples' respective fractions of female patients were $53 \%$ versus $50 \%$ (Fisher $\mathrm{p}=0.905$ ) and their median BMI was $28.9 \mathrm{~kg} / \mathrm{m}^{2}\left(20-38 \mathrm{~kg} / \mathrm{m}^{2}\right)$ versus $26.6 \mathrm{~kg} / \mathrm{m}^{2}(21-$ $39 \mathrm{~kg} / \mathrm{m}^{2}$, Wilcoxon $\left.\mathrm{p}=0.367\right) .77 \%$ of the patients of the posterior group and $67 \%$ of the direct laterally implanted patients were married, $63 \%$ of the patients in both groups were employed.

\section{PRIMARY OBJECTIVE AND SAMPLE SiZE CALCULATION}

Main clinical outcome measures were pain, function and range of movement evaluated by means of the
Harris hip rating system (HHS). 11 The primary clinical endpoint of this investigation was defined as the intraindividual increase at the 12 weeks assessment versus its preoperative level. Sample size calculation was based on the assumption of a minimum clinically relevant difference of 5 points and a standard deviation of 6 points in the HHS total score. A total of 24 patients had to be enrolled per treatment to achieve a statistical power of at least $80 \%$ in detection of the above mentioned effect size at a 5\% statistical significance level by means of a two sample t-test. Assuming a $20 \%$ drop out rate a net sample size of 30 patients per treatment was required.

\section{SECONDARY OBJECTIVES}

The Western Ontario and McMaster Universities Osteoarthritis Index (WOMAC), the Short Form-36 (SF36) questionnaire and patient activity assessed by means of the Tegner activity score were measured to provide additional secondary patient-related endpoints $[3,22,23]$. Both, the WOMAC total score as well as its constituent sub dimensions (pain, stiffness, daily life activities) were transformed into a utility estimate with range between $0-100 \%$ (optimum rating); the physical and mental scale estimates derived from the SF-36 interviews were analyzed accordingly.

The total range of motion and leg length discrepancy assessed by means of blocks with different thicknesses, which were placed under each patient's foot until the pelvis leveled served as additional clinical outcome measurements.

Early postoperative function was compared at the 7 th day after surgery by means of the walking time over $25 \mathrm{~m}$, the highest possible hip flexion in standing position and the median step length at a $25 \mathrm{~m}$ gait course (length of gait course divided through number of steps).

\section{ADVERse OUtCOMES}

Adverse outcomes were measured by investigation of the rate of intra- and postoperative complications (periprosthetic fracture, nerve palsy, wound infection, deep thrombosis or pulmonary embolism, dislocation) and the rate of positive Trendelenburg signs.

\section{OPERATIVE DETAILS}

Operative details (operative time, blood transfusion, length of incision) were recorded.

\section{SURGICAL TECHNIQUE}

Surgery was performed under general anesthesia by two experienced surgeons (11 posterior, 10 direct lateral implantations by KPG and 19 posterior, 20 direct lateral implantations by WCW). Preoperatively, all patients received one dose of an intravenous cephalosporin.

The posterior approach entailed a curved incision centered on the greater trochanter in lateral decubitus position of the patient. The fascia lata was incised in line of the skin incision and the fibers of the gluteus 
maximus were split by blunt dissection. The short external rotators were then detached close to their femoral insertion leaving one centimeter of muscle tissue of the quadratus femoris at the dorsal aspect of the greater trochanter for re-attachment. The posterior hip capsule was incised and preserved. After implantation, the posterior capsule was re-attached on the greater trochanter together with the short external rotators and the wound was closed in layers [17].

The direct lateral approach entailed a longitudinal skin incision centered over the greater trochanter in supine position. The tractus iliotibialis and the gluteal fascia were divided in the line of the skin incision. The anterior part of the gluteus medius and minimus insertion was incised down to the bone, prolonged distally through the vastus lateralis in a curved line to spare some tendinous tissue at the greater trochanter for reattachment. The anterior hip capsule was excised. After implantation, the tendinous tissue was re-attached at the greater trochanter and the wound was closed in layers $[2,10]$.

\section{IMPLANTS}

All patients were implanted with a cementless press-fit cup, cementless straight stem and a $28 \mathrm{~mm}$ metal-onmetal (in cases of metal allergy ceramic-on-ceramic) articulation (Fitmore or Allofit cup, CLS stem, Metasul or Cerasul bearing, Zimmer Ltd., Warsaw, US). 12 cups, 6 in each group, were fixed additionally with 1 to 3 screws.

\section{Postoperative Management}

Low molecular heparin (0.2-0.6 ml fraxiparine per day, weight-adapted, GlaxoSmithKline GmbH, Germany) was used for thromboprophylaxis until re-mobilization, at least for three weeks. $150 \mathrm{mg}$ diclophenac per day was used for two weeks in order to prevent the formation of heterotopic bone.

Walking training was started on the first postoperative day, with full weight-bearing allowed. All patients underwent a standardized physiotherapy program until hospital discharge at the seventh postoperative day.

Following discharge, all patients trained walking under full weight-bearing with two crutches and received physiotherapy at an individual basis. During the first four weeks, hip flexion was limited to $90^{\circ}$ and forced internal as well as external rotation was not allowed.

Four weeks after surgery all patients were admitted to a cooperative rehabilitation department, where they underwent a standardized rehabilitation program for three weeks.

\section{RADIOGRAPHIC ANALYSIS}

All patients had anteroposterior radiographs of the pelvis taken pre-, postoperatively and at the three months recall. Radiographs were studied by an independent observer (LS). Component position was analyzed using the abduction angle of the cup and the varus-valgus position of the stem. Heterotopic ossification was graded according to the Brooker classification [4].

\section{STATISTICAL ANALYSIS}

All numerical and graphical evaluations were performed by an independent biometrician (F.K.) by means of the software SPSS (release 12.0 for Windows). Data description was based on medians, minima and maxima for continuous endpoints and on absolute and relative frequencies for categorical endpoints. The graphical representation of continuous data was based on nonparametric box whisker plots, accordingly. The description of intraindividual comparisons in continuous endpoints at different assessment times was based on the distribution of intraindividual differences. For significance evaluation of these intraindividual comparisons at continuous endpoints, the Sign test was applied. For the comparison of sub samples the two sample Wilcoxon test was applied for continuous endpoints (such as the primary clinical endpoint) and the Fisher test for categorical endpoints. Results of significance tests were summarized by means of $\mathrm{p}$-values.

Analysis of the primary research hypothesis of this investigation was therefore based on the descriptive comparison of the primary clinical endpoint (HHS increase after 12 weeks) between the posterior and lateral treatment group as well as on the derivation of a two-sided Wilcoxon test $\mathrm{p}$-value (as suggested by the Statistical Analysis Plan of the trial). Note that this confirmatory analysis strategy deviates from the t-test assumption introduced into the sample size calculation: since the occurrence of statistical outliers could not be excluded during the preparation of the trial's statistical analysis plan, the confirmatory analysis directly focussed on the application of the two sample Wilcoxon test. Although the latter shows a gradually smaller statistical power when compared to its two sample t-test analogue, the more robust handling of possible outliers was considered as a dominating advantage.

If further significance tests were performed in the context of additional exploratory analyses, the resulting $\mathrm{p}$-values were not adjusted for multiplicity due to the exploratory character of these analyses; a p-value $<0.05$ therefore indicates locally statistical significance.

\section{RESULTS}

\section{OPERATIVE DETAILS}

Operative time (cut-suture-time) was significantly longer in the posterior group (76.5 minutes [59- 105 min] versus 67 minutes $[47-87 \mathrm{~min}]$, Wilcoxon $\mathrm{p}<0.001)$. The groups did not differ in the incision length measured three months after surgery $(16 \mathrm{~cm}$ $[11-23 \mathrm{~cm}]$ in the posterior group versus $15 \mathrm{~cm}[11-$ $19 \mathrm{~cm}$ ] direct lateral, Wilcoxon $\mathrm{p}=0.339)$. Postoperative blood transfusion (one unit of $400 \mathrm{ml}$ ) was needed in $23 \%$ per group.

\section{PRIMARY CLINICAL ENDPOINT}

Patients implanted via a posterior approach started pre-operatively with a median total HHS score of 46 
points $(29-64)$ slightly worse than the patients in the directly lateral group (median HHS 50 pts. [29 - 76]), but showed a slightly superior outcome with in median 83 points $(68-89)$ versus 77 points $(65-82)$ at the three months recall assessment (Table 1, Fig. 1). However, the overall HHS of both treatment groups did not differ significantly at any assessment time (Wilcoxon $\mathrm{p}=0.307$ pre-operatively, $\mathrm{p}=0.724$ at four weeks, $\mathrm{p}=0.618$ at six weeks and $\mathrm{p}=0.075$ after three months; Table 1).

Median total HHS score profiles increased in both groups significantly over the observation period (respective sign test $\mathrm{p}<0.001)$. Patients in the posterior group improved by in median 7 points $(-5-15)$ during the first four weeks, 18 points $(8-27)$ during six weeks and 30 points $(20-48)$ during the three months period (Table 1, Fig. 2). The corresponding median intraindividual HHS increases in the directly lateral group were 10 points $(-6-18), 14$ points $(3-$ $28)$ and 25 points $(-15-36)$. The treatment groups did not differ significantly at any assessment time (Wilcoxon $\mathrm{p}=0.967, \mathrm{p}=0.451, \mathrm{p}=0.115$, respectively; Table 1), in particular the confirmatory research hypothesis of the investigation, which concentrated on the three months HHS increase, could not be confirmed significantly.

Harris Hip sub scores (pain, function and movement) also showed no significant differences between the treatment groups (for example, respective Wilcoxon $\mathrm{p}$-values for pain scores were $\mathrm{p}=0.378, \mathrm{p}=0.336$, $p=0.492, p=0.259)$. However, the groups showed a slightly different tendency in function improvement: in the posterior group, the function sub score rose from in median 28 points $(13-41)$ preoperatively over 20 points $(13-28)$ after four weeks and 26 points (17 - 33) after six weeks to 36 points $(20-47)$ at three months slightly superiorly in versus 31 points $(12-$ 39), 18 points $(8-29), 24$ points $(17-34)$ and 32 points $(14-41)$ in the direct lateral group (Wilcoxon $\mathrm{p}$ $=0.264, \mathrm{p}=0.118, \mathrm{p}=0.650$ and $\mathrm{p}=0.073$, respectively). On the contrary, movement scores and total range of motion turned out slightly better in the directly lateral group with median scores of $7,7,7$ points and 9 points versus 7, 7, 7 and 8 points after posterior surgery (Wilcoxon $\mathrm{p}=0.646, \mathrm{p}=1.000, \mathrm{p}=$ 0.287 and $\mathrm{p}=0.071$, respectively) and in median $210^{\circ}$ $\left(150^{\circ}-260^{\circ}\right)$ versus $190^{\circ}\left(145^{\circ}-255^{\circ}\right.$, Wilcoxon $\mathrm{p}=$ $0.105)$.

\section{SECONDARY OBJECTIVES}

Early postoperative function at the seventh day after surgery:

Median walking times at the $25 \mathrm{~m}$ gait course did not differ between both groups (posterior group: 35 seconds [27 - $57 \mathrm{sec}]$, direct lateral group: $38 \mathrm{sec}$ [28 - 56 sec], Wilcoxon $\mathrm{p}=0.361)$. Patients after posterior surgery had a slight, but not significantly, larger resulting step length of $60 \mathrm{~cm}(45-66 \mathrm{~cm})$ versus $54 \mathrm{~cm}$ $(43-71 \mathrm{~cm})$ of the patients with a direct lateral approach (Wilcoxon $\mathrm{p}=0.098$ ). While total range of motion was significantly better after direct lateral implantation $\left(120^{\circ}\left[95-150^{\circ}\right]\right.$ versus $110^{\circ}\left[90-145^{\circ}\right]$, Wilcoxon $\mathrm{p}=0.037$, active hip flexion in standing po- sition showed no difference between the groups (posterior group: $70^{\circ}\left[25-90^{\circ}\right]$, direct lateral group: $70^{\circ}$ $\left[40-90^{\circ}\right.$, Wilcoxon $\left.\mathrm{p}=0.722\right)$.

\section{Western Ontario and McMaster Universities Os-} teoarthritis Index:

Patients in the posterior group had slightly, but not significantly smaller scores before surgery, and somewhat, but not significantly better results after three months: Median WOMAC profiles increased in both groups significantly over the observation period (respective sign test $\mathrm{p}<0.001$ ) from $38 \%$ versus $43 \%$ before surgery over $66 \%$ versus $67 \%$ four weeks afterwards, $72 \%$ versus $76 \%$ six weeks after surgery to $87 \%$ versus $81 \%$ in the three months recall. The samples did not differ significantly at any assessment time (Wilcoxon $\mathrm{p}=0.109, \mathrm{p}=0.961, \mathrm{p}=0.703, \mathrm{p}=0.359$, respectively). Patients in the posterior group intraindividually improved by in median $28 \%(14-38 \%)$ during four weeks, $38 \%(26-49 \%)$ during six weeks, and $49 \%(34-58 \%)$ during three months. In contrast, the three months improvement in the directly lateral group was estimated 30\% $(19-47 \%)$ in median and found significantly smaller (Wilcoxon $p=0.007$ ) compared to the three months increase in the posterior group. The four and six weeks changes after directly lateral surgery were $15 \%(2-34 \%)$ and $30 \%(19-$ $47 \%$ ), respectively.

Concerning the WOMAC sub scores related to pain, stiffness and daily life activities, the posterior implanted patients showed gradually lower scores before surgery and ended with higher scores in the three months assessment for each sub dimension (Table 2). For example the pain sub score rose from $40 \%$ before posterior surgery to $91 \%$ three months afterwards versus an increase from $47 \%$ to $88 \%$ after direct lateral surgery (Wilcoxon $\mathrm{p}=0.383$ and 0.182 , respectively). However, the treatment groups did not differ significant in any of the sub scores (Table 2).

\section{Short-Form 36 Questionnaire:}

SF-36 mental and physical scales showed no differences at any assessment point (Table 3): The physical scale score increased from $26 \%$ before to $50 \%$ after posterior surgery and from $28 \%$ to $49 \%$ after direct lateral implantation (Wilcoxon $\mathrm{p}=0.515$ and $\mathrm{p}=$ 0.426 , respectively), the corresponding mental scale scores rose from $60 \%$ to $78 \%$ and from $53 \%$ to $69 \%$ (Wilcoxon $\mathrm{p}=0.455$ and $\mathrm{p}=0.439$, respectively).

\section{Patient activity:}

Patient activity levels according to the Tegner activity score were preoperatively slightly lower, and three months afterwards gradually higher in the posterior group with median increases of 2.5 points versus 1 point after direct lateral approach, but did not significantly differ from those in the direct lateral group (Wilcoxon $\mathrm{p}=0.083$ ).

\section{Total range of motion:}

Three months postoperatively, patients after the direct lateral approach showed a total range of motion of in median $210^{\circ}\left(150^{\circ}-260^{\circ}\right)$ versus $190^{\circ}\left(145^{\circ}-255^{\circ}\right)$ of patients in the posterior group (Wilcoxon $\mathrm{p}=0.105$ ). 
Table 1. Medians, minima and maxima for the distribution of total HHS scores before and four, six, 12 weeks after posterior versus direct lateral THA; medians of the respective intraindividual changes' distributions (post - preoperative); p-values derived from two sample Wilcoxon test at each assessment time, respectively.

\begin{tabular}{|c|c|c|c|c|}
\hline \multicolumn{5}{|c|}{ total HHS score } \\
\hline approach & preoperative & four weeks & six weeks & three months \\
\hline posterior & $46(29-64)$ & $53(44-59)$ & $63(58-77)$ & $83(68-89)$ \\
\hline direct lateral & $50(29-76)$ & $55(35-72)$ & $62(44-80)$ & $77(65-82)$ \\
\hline p-value & 0.307 & 0.724 & 0.618 & 0.075 \\
\hline \multicolumn{5}{|c|}{ change in total HHS score (post - preoperative) } \\
\hline approach & & four weeks & six weeks & three months \\
\hline posterior & & $7(-5 ; 15)$ & $18(8 ; 27)$ & $30(20 ; 48)$ \\
\hline direct lateral & & $10(-6 ; 18)$ & $14(3 ; 28)$ & $25(15 ; 36)$ \\
\hline $\mathrm{p}$-value & & 0.967 & 0.451 & 0.115 \\
\hline
\end{tabular}

Table 2. Medians, minima and maxima for the distribution of WOMAC sub scores for pain, stiffness and daily life activities (range $0-100 \%, 100 \%=$ optimum rating) before and four, six, 12 weeks after posterior versus direct lateral approach; p-values derived from two sample Wilcoxon test at each assessment time, respectively.

\begin{tabular}{|c|c|c|c|c|c|}
\hline $\begin{array}{l}\text { WOMAC } \\
\text { sub scores } \\
{[0-100 \%]}\end{array}$ & approach & preoperative & four weeks & six weeks & three months \\
\hline pain & $\begin{array}{c}\text { posterior } \\
\text { direct lateral } \\
\mathrm{p} \text {-value }\end{array}$ & $\begin{array}{c}40(0-100) \\
47(0-80) \\
0.383\end{array}$ & $\begin{array}{c}73(24-100) \\
78(0-100) \\
0.571\end{array}$ & $\begin{array}{c}80(36-100) \\
84(0-100) \\
0.870\end{array}$ & $\begin{array}{c}91(34-100) \\
88(0-100) \\
0.182\end{array}$ \\
\hline stiffness & $\begin{array}{c}\text { posterior } \\
\text { direct lateral } \\
\text { p-value }\end{array}$ & $\begin{array}{c}38(0-80) \\
45(0-80) \\
0.313\end{array}$ & $\begin{array}{c}70(25-95) \\
75(10-100) \\
0.128\end{array}$ & $\begin{array}{c}80(35-100) \\
80(20-100) \\
0.827\end{array}$ & $\begin{array}{c}85(30-100) \\
80(20-100) \\
0.231\end{array}$ \\
\hline daily activity & $\begin{array}{c}\text { posterior } \\
\text { direct lateral } \\
\text { p-value }\end{array}$ & $\begin{array}{c}35(0-67) \\
47(0-76) \\
0.055\end{array}$ & $\begin{array}{c}63(14-92) \\
62(0-90) \\
0.799\end{array}$ & $\begin{array}{c}72(25-95) \\
74(0-96) \\
0.773\end{array}$ & $\begin{array}{c}84(45-97) \\
81(0-100) \\
0.476\end{array}$ \\
\hline
\end{tabular}

Table 3. Medians, minima and maxima for the distribution of SF-36 physical and mental scale (range $0-100 \%, 100 \%=$ optimum rating) before and four, six, 12 weeks after posterior versus direct lateral approach; p-values derived from two sample Wilcoxon test at each assessment time, respectively.

\section{SF-36 physical scale [range $0-100 \%$ ]}

\begin{tabular}{r|c|c|c|c}
\hline approach & preoperative & four weeks & six weeks & three months \\
posterior & $26(8-64)$ & $36(13-68)$ & $44(21-91)$ & $50(19-92)$ \\
direct lateral & $28(9-70)$ & $33(12-61)$ & $40(17-70)$ & $49(14-91)$ \\
p-value & 0.515 & 0.359 & 0.879 & 0.426 \\
\hline
\end{tabular}

SF-36 mental scale [range $0-100 \%$ ]

\begin{tabular}{c|c|c|c|c}
\hline approach & preoperative & four weeks & six weeks & three months \\
posterior & $60(22-89)$ & $62(28-82)$ & $68(34-89)$ & $78(20-94)$ \\
direct lateral & $53(12-87)$ & $48(12-86)$ & $54(22-91)$ & $69(26-90)$ \\
p-value & 0.455 & 0.085 & 0.175 & 0.439 \\
\hline
\end{tabular}



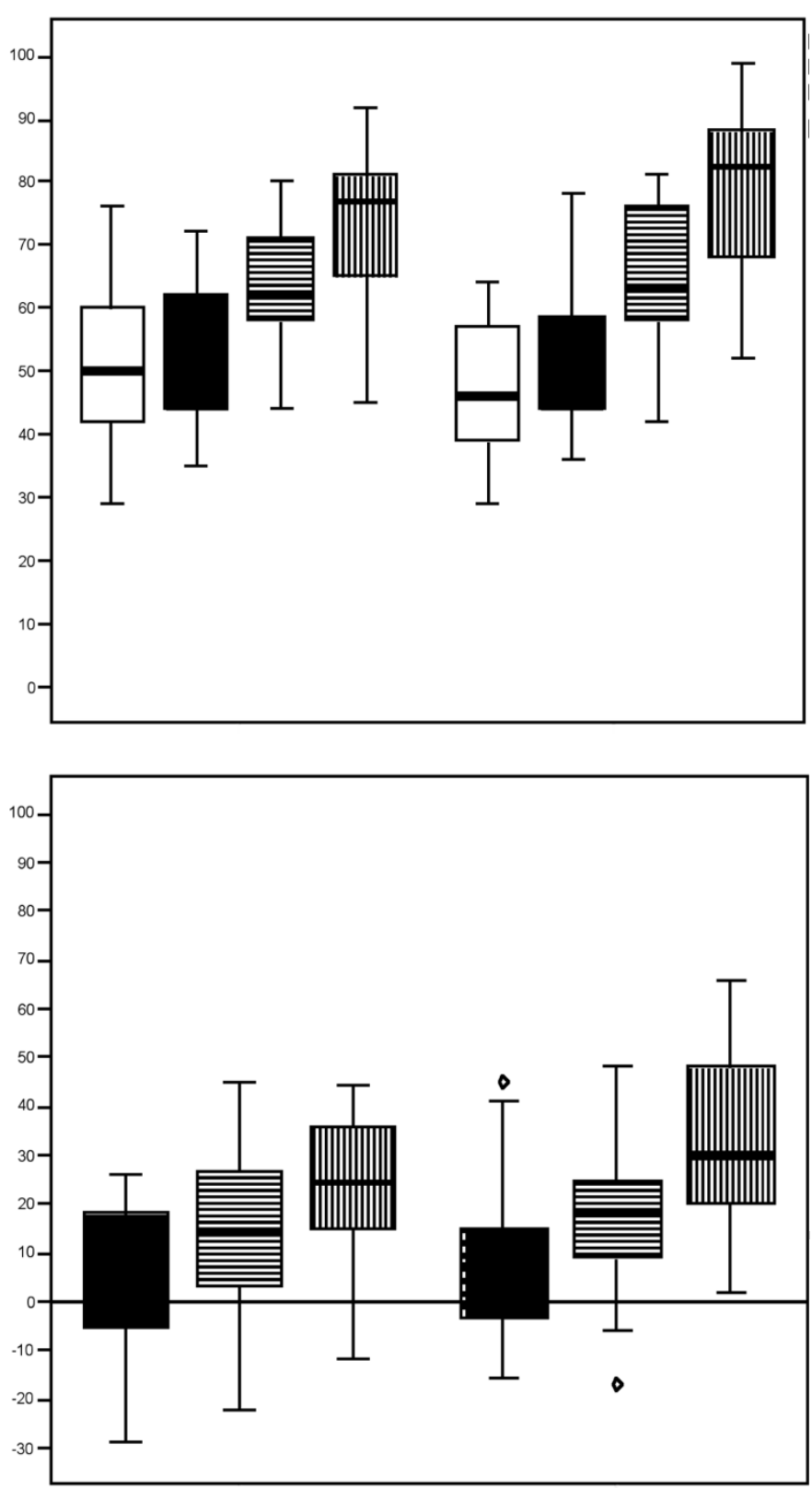

$\square$ HHS, before surgery

HHS, four weeks after surgery

E HHS, six weeks after surgery W $\begin{aligned} & \mathrm{HHS} \text {, three months after } \\ & \text { surgery }\end{aligned}$
Fig. 1. Box plots for the distribution of Harris Hip Score profiles four, six and 12 weeks after THA via posterior versus direct lateral approach (horizontals indicate medians and quartiles, verticals indicate minimum and maximum observations).

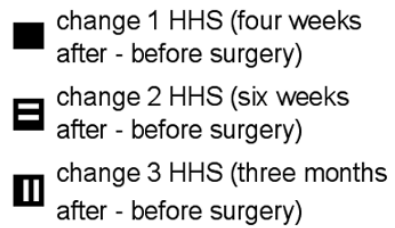

Fig. 2. Box plots for the distribution of intraindividual Harris Hip Score changes four, six and 12 weeks after THA via posterior versus direct lateral approach (horizontals indicate medians and quartiles, verticals indicate minimum and maximum observations).

\section{Leg length discrepancy:}

Preoperatively, $50 \%$ of the patients in both groups had no leg length discrepancy. In 40\% (posterior approach) versus $43 \%$ (direct lateral approach) a shortening of the involved side, ranging between $0.5-2 \mathrm{~cm}$ was observed. Three months postoperatively, $27 \%$ of the patients with a posterior approach showed no discrepancy versus $43 \%$ of the direct lateral group. 50\% of the patients in the posterior group had a longer leg $(0.5-2 \mathrm{~cm}$, one patient [3\%] over $2 \mathrm{~cm})$ on the implanted side versus $43 \%$ in the direct lateral group. The occurrence rates of leg length discrepancies did not statistically differ (Fisher $\mathrm{p}=0.363$ ).

\section{Adverse Outcomes}

\section{Complications:}

In each group one non-dislocated fracture of the greater trochanter occurred intra-operatively without further complication under full weight-bearing. Nerve lesions were not recorded in any of the groups. Two patients in the direct lateral group developed a superficial wound infection, which resolved spontaneously. Whereas none of the direct laterally implanted patients developed a clinically diagnosed deep vein thrombosis, in two cases of the posterior group a thrombosis was observed.

\section{Dislocations:}

One posteriorly implanted patient dislocated his hip at the fifth postoperative day. After closed reduction he was re-mobilized with full weight-bearing without further complications.

\section{Trendelenburg Sign:}

While $33 \%$ of the patients in each group showed positive or indifferent Trendelenburg signs before surgery, only $13 \%$ of the posteriorly implanted patients did so at the three months follow-up examination. Patients in the direct lateral group had at the borderline of signifi- 
cance more frequent positive or indifferent Trendelenburg signs (37\%) at the three months recall (Fisher $\mathrm{p}=$ 0.072).

\section{RADIOGRAPHIC RESULTS}

The median abduction angle of the cup was not significantly different between both groups: Posteriorily implanted hips showed a median abduction angle of $48^{\circ}\left(34^{\circ}-58^{\circ}\right)$ in contrast to $45^{\circ}\left(32^{\circ}-58^{\circ}\right)$ in the direct lateral group (Wilcoxon $\mathrm{p}=0.477$ ). Two stems of the direct lateral group were graded to be in slight varus. All other femoral components were graded to be in neutral position (Fisher $\mathrm{p}=0.492$ ).

Heterotopic bone formation. Three months postoperatively, heterotopic ossification was present in 7\% (each grade II) of the patients with a posterior approach. In contrast, $30 \%$ (27\% grade I, 3\% grade II) of the patients with a direct lateral approach showed heterotopic ossifications (Fisher $\mathrm{p}=0.061$ ).

\section{Discussion}

Many different surgical approaches to the hip have been described. Currently, the principal methods for THA are the posterior and direct lateral approaches [5, 15]. We studied these since they are the two most commonly performed surgical approaches in our unit and both provide adequate exposure for implantation. The relative merits of these approaches are debated, although no study has conclusively demonstrated an advantage of one over the other. Many of the trial reports published involved only small numbers of participants with limited reporting of outcome measures and a poor level of methodological rigor [12]. To our knowledge only Pascarel et al. (1989) randomly compared 63 patients after a posterior approach with 63 patients after a direct lateral approach [19]. They found a lower dislocation rate, but a higher rate of gluteal deficiencies (33\% versus 17\%) with the direct lateral approach. But hip replacements in their investigation were performed for several reasons (hip fractures, avascular necrosis of the femoral head, DDH and osteoarthritis as well) and no specific data were available for patients with osteoarthritis alone.

To the authors' knowledge this is the first randomized controlled trial in osteoarthritis patients with the hypothesis of a better short-term functional outcome of THR through the posterior in contrast to the direct lateral approach.

In general we could not confirm our hypothesis of a superior short-term functional improvement after posterior surgical approach due to minor violation of the hip abductors. Despite the strong inclusion criteria used (only Charnley grade A patients, standardized rehabilitation), we were not able to show a significant difference between both approaches in the Harris hip rating system, which served as primary objective of our study.

As indicated in the Methods section, this investigation was designed to achieve a statistical power of at least $80 \%$ in the detection of a 5 point difference in the HHS changes between both treatment groups. The actual median HHS changes were indeed 30 points af- ter posterior approach versus 25 points after direct lateral approach, but were complemented by rather unexpectedly large interquartile ranges (Fig. 2). Note, that the sample size calculation does not only involve the sample difference in median, but also assumptions on the underlying sample variations, which were underestimated during the planning phase of the trial: A substantial heterogeneity was observed between and within both patient cohorts, which could hardly be considered during the planning phase of the trial. The latter might have been caused by varying motivations among our patients (for example, due to employment or family status). Despite randomization patients in the posterior approach group showed not significantly but somewhat lower pre-operative score levels, what might have influenced the pre-/postoperative differences. Another bias might have been introduced by the rather short observation period. Not all patients may have reached their optimal functional status again after 12 weeks. The authors therefore intend to prolong this investigation for one additional postoperative year.

However, most functional and psychometric endpoints investigated showed a consistent tendency of a slightly better short-term result in patients implanted via the posterior approach. This patient group showed at the border of significance better Harris hip scores at the three months recall. Additionally, a slightly - although not significantly better result could be observed in most secondary endpoints (i.e. walking time, stride length and patient activity) after THR through the posterior approach. The improvement of the WOMAC index between preoperative and three months postoperative assessments was even significantly better in patients with a posterior approach and this patient group showed less positive Trendelenburg signs three months postoperatively. This outcome measure was found to slightly favor the posterior approach as having fewer participants with postoperative Trendelenburg gait also by other investigators [1, 14, 21]. Damage of the superior gluteal nerve or failure to re-attach the muscles during direct lateral approach are debated as possible reasons. In contrast, direct lateral approach counted with a significant shorter operating time, fewer clinically relevant deep vein thromboses and no versus one dislocation. However, the missing significance in the primary endpoint of our investigation prevents definite conclusions from being drawn. Other factors, like patient related factors, experience of the surgeon and/or implant type may have a greater influence on the result.

In summary, we could not confirm our hypothesis of a superior short-term functional improvement of patients implanted with a THR via the posterior versus the direct lateral surgical approach. The difference between both patient groups in intraindividual changes between pre- and three months postoperative HHS, which served as our primary endpoint, did not reach statistical significance. We could observe, however, a consistent tendency of better functional results in most of functional and psychometric secondary endpoints. But, however, these findings are not clinical relevant in our opinion and are contrasted by the significant shorter operating time of the direct lateral approach. To make definitive conclusions all clinical rele- 
vant factors (i.e. mid- to long-term function, satisfaction, complication rates and long-term survival) have to be taken into account. We intend to prolong our study for one postoperative year.

Acknowledgement: The authors would like to thank Mrs Heike Voigt and Mrs Claudia Wojciechowski for assistance in data management and analysis, Ms Stephanie Reinhart (MD) for assistance in clinical data acquisition, and Mr Patrick Hunt for a native speaker revision of the manuscript.

Conflict of Interests statement: The authors have no commercial or political interests in the clinical products and findings presented in this manuscript. The investigation was not granted.

\section{REFERENCES}

1. Baker AS, Bitounis VC. Abductor function after total hip replacement. An electromyographic and clinical review. J Bone Joint Surg Br. 1989;71(1):47-50.

2. Bauer R, Kerschbaumer F, Poisel S, Oberthaler W. The transgluteal approach to the hip joint. Arch Orthop Trauma Surg. 1979;95(1-2):47-49.

3. Bellamy N. The WOMAC Knee and Hip Osteoarthritis Indices: development, validation, globalization and influence on the development of the AUSCAN Hand Osteoarthritis Indices. Clin Exp Rheumatol. 2005;23(5 Suppl 39):S148-153.

4. Brooker AF, Bowerman JW, Robinson RA, Riley LH, Jr. Ectopic ossification following total hip replacement. Incidence and a method of classification. J Bone Joint Surg Am. 1973;55(8):1629-1632.

5. Bystrom S, Espehaug B, Furnes O, Havelin LI. Femoral head size is a risk factor for total hip luxation: a study of 42,987 primary hip arthroplasties from the Norwegian Arthroplasty Register. Acta Orthop Scand. 2003;74(5): 514-524.

6. Charnley J. The long-term results of low-friction arthroplasty of the hip performed as a primary intervention. J Bone Joint Surg Br. 1972;54(1):61-76.

7. Comstock C, Imrie S, Goodman SB. A clinical and radiographic study of the "safe area" using the direct lateral approach for total hip arthroplasty. J Arthroplasty. 1994;9(5):527-531.

8. Crowe JF, Mani VJ, Ranawat CS. Total hip replacement in congenital dislocation and dysplasia of the hip. J Bone Joint Surg Am. 1979;61(1):15-23.

9. Downing ND, Clark DI, Hutchinson JW, Colclough K, Howard PW. Hip abductor strength following total hip arthroplasty: a prospective comparison of the posterior and lateral approach in 100 patients. Acta Orthop Scand. 2001;72(3):215-220

10. Hardinge K. The direct lateral approach to the hip. J Bone Joint Surg Br. 1982;64(1):17-19.
11. Harris WH. Traumatic arthritis of the hip after dislocation and acetabular fractures: treatment by mold arthroplasty. An end-result study using a new method of result evaluation. J Bone Joint Surg Am. 1969;51(4):737-755.

12. Jolles BM, Bogoch ER. Posterior versus lateral surgical approach for total hip arthroplasty in adults with osteoarthritis. Cochrane Database Syst Rev. 2006;3:CD003828.

13. Kellgren JH, Lawrence JS. Radiological assessment of osteo-arthrosis. Ann Rheum Dis. 1957;16(4):494-502.

14. Kenny P, O'Brien CP, Synnott K, Walsh MG. Damage to the superior gluteal nerve after two different approaches to the hip. J Bone Joint Surg Br. 1999;81(6):979-981.

15. Kwon MS, Kuskowski M, Mulhall KJ, Macaulay W, Brown TE, Saleh KJ. Does surgical approach affect total hip arthroplasty dislocation rates? Clin Orthop Relat Res. 2006;447:34-38.

16. Li E, Meding JB, Ritter MA, Keating EM, Faris PM. The natural history of a posteriorly dislocated total hip replacement. J Arthroplasty. 1999;14(8):964-968.

17. Moore A. T. In: Surgeons AAoO, ed. Intructional course lectures. Vol 16. St. louis: CV Mosby; 1959.

18. Mulliken BD, Rorabeck CH, Bourne RB, Nayak N. A modified direct lateral approach in total hip arthroplasty: a comprehensive review. J Arthroplasty. 1998;13(7):737747.

19. Pascarel X, Dumont D, Nehme B, Dudreuilh JP, Honton JL. [Total hip arthroplasty using the Hardinge approach. Clinical results in 63 cases]. Rev Chir Orthop Reparatrice Appar Mot. 1989;75(2):98-103.

20. Paterno SA, Lachiewicz PF, Kelley SS. The influence of patient-related factors and the position of the acetabular component on the rate of dislocation after total hip replacement. J Bone Joint Surg Am. 1997;79(8):1202-1210.

21. Ramesh M, O'Byrne JM, McCarthy N, Jarvis A, Mahalingham $\mathrm{K}$, Cashman WF. Damage to the superior gluteal nerve after the Hardinge approach to the hip. J Bone Joint Surg Br. 1996;78(6):903-906.

22. Tegner Y, Lysholm J. Rating systems in the evaluation of knee ligament injuries. Clin Orthop Relat Res. 1985(198): 43-49.

23. Ware JE, Jr., Sherbourne CD. The MOS 36-item shortform health survey (SF-36). I. Conceptual framework and item selection. Med Care. 1992;30(6):473-483.

Received: November 10, 2008 / Accepted: April 2, 2009

Address for correspondence:

Dr. med. Wolf-Christoph Witzleb

Forum Gesundheit Dresden

Leipziger Str. 40; D-01307 Dresden (Germany)

Tel.: 0049 (0)351 4583323

Fax.: 0049 (0)351 4584376

Email:dr.witzleb@forum-gesundheit-dresden.de 